\title{
Getting ready for fieldwork education! - Creation of a skill profile for fieldwork educators in occupational therapy Fit für die Praktikumsanleitung? - Erstellung eines Anforderungsprofils für ergotherapeutische Praxislehrende
}

\author{
Elfriede Galler ${ }^{1 *}$, Renate Ruckser-Scherb² \\ ${ }^{1}$ SKA-RZ Gröbming der Pensionsversicherungsanstalt, \\ 8960 Gröbming, Austria \\ *galler-ergotherapie@aon.at \\ ${ }^{2}$ Fachhochschule Gesundheitsberufe $\mathrm{OÖ} \mathrm{GmbH}$, \\ Studiengang Ergotherapie, 4020 Linz, Austria
}

Received 12 April 2018, accepted 24 July 2018

\begin{abstract}
Owing to ongoing changes in the working world and the resulting changing requirements of university graduates, requirements for teachers are continuously changing. Although this is currently being taken into account at universities, there is a need to take action in the area of fieldwork education.

The aim was to create a skill profile for fieldwork educators in occupational therapy, thus providing a basis for fieldwork educators, universities, professional associations, and employers. This reflects on existing measures and generates new measures to facilitate quality improvement in the area of fieldwork education.

Requirements were evaluated in two focus groups with a total of 14 practical training experts. Data were analysed within the scope of a qualitative content analysis and summarized in one profile.

A total of 18 professional, methodical, social, and personal requirements were identified. Fieldwork educators are able to organise, take responsibility, have expertise, and prepare contents adapted to the needs of individual students. They guide, supervise, give feedback to students in a formative way, thereby assessing and supporting their personal development. Moreover, as communicators, they have a stable personality, are open for further development, flexible, and reflective.

In order to meet these requirements, fieldwork educators must be fully committed. Moreover, the professional association needs to create a role model and provide task-specific cross-university training. It also requires the support of universities who are called upon to increase networking and communication with all parties involved and the support of the employers who need to provide additional time resources.
\end{abstract}

\begin{abstract}
Durch den permanenten Wandel in der Arbeitswelt und den daraus resultierenden sich ändernden Anforderungen an die Absolventinnen und Absolventen einer Hochschule verändern sich auch die Voraussetzungen für Lehrende. Das wurde bislang am Standort Fachhochschule berücksichtigt, für die Lehre in der realen Berufswelt herrscht noch Handlungsbedarf.

Ziel war das Erstellen eines Anforderungsprofils für die ergotherapeutische Praktikumsanleitung. Dieses bietet sowohl für die Praktikumsanleitung selbst als auch für die Fachhochschulen, den Berufsverband und die Dienstgeber eine Grundlage zum Reflektieren bereits bestehender und zum Schaffen neuer, die Qualität der Praktikumsanleitung unterstützender Maßnahmen. In zwei Fokusgruppen von insgesamt 14 Expertinnen und Experten in der ergotherapeutischen Praktikumsanleitung wurden Anforderungen erhoben. Die Daten wurden im Rahmen einer qualitativen Inhaltsanalyse ausgewertet und in einem Anforderungsprofil zusammengefasst.

Es konnten 18 Anforderungen aus dem fachlich-methodischen, sozialen und personalen Bereich identifiziert werden. Praxislehrende organisieren, übernehmen Verantwortung, besitzen Fachwissen und können dieses auf den Lerntypus und das Lernniveau abgestimmt aufbereiten. Sie leiten an, supervidieren, geben formatives Feedback, beurteilen und unterstützen die Studierenden in deren Persönlichkeitsentwicklung. Darüber hinaus weisen sie als Kommunikatoren/-innen eine stabile Persönlichkeit auf, sind offen für Weiterentwicklung, flexibel und reflektiert.

Damit die Praktikumsanleitung die Anforderungen erfüllen kann, braucht es neben Engagement ihrerseits auch die Unterstützung des Berufsverbandes über das Erstellen eines Rollenbildes und der Bereitstellung von aufgabenspezifischen fachhochschulübergreifenden Weiterbildungsmöglichkeiten. Ebenso bedarf es der Unterstützung der Fachhochschulen über eine vermehrte Vernetzung und Kommunikation mit allen Beteiligten sowie der Unterstützung der Dienstgeber über die Bereitstellung von Zeitressourcen.
\end{abstract}

\section{Keywords}

teaching quality in higher education - fieldwork education - pedagogical suitability - skill profile - person-environment fit

\section{Keywords}

Qualität in der Hochschullehre - Berufspraktikum - Pädagogische Eignung - Anforderungsprofil - Person-Environment Fit 


\section{EINLEITUNG}

Die Unterzeichnung des Bologna-Abkommens 1999 zog einen grundlegenden Reformprozess im europäischen Hochschulwesen nach sich (vgl. Schriewer, 2006). Die dadurch initiierte Akademisierung der Ausbildung in den gehobenen medizinisch-technischen Diensten auf Fachhochschulebene (vgl. MTD-Gesetz 2005 § 3, Abs. 4-6) gestaltete sich im Bereich der Ergotherapie als große Herausforderung. Unter anderem war und ist es erforderlich, die Lehre nach den Richtlinien der BolognaErklärung so zu konzipieren, dass die Absolventinnen und Absolventen neben der Berufsbefähigung und der wissenschaftlichen Professionalität auch den in letzter Zeit ständig steigenden Anforderungen hinsichtlich Flexibilität und Mobilität im Berufsfeld gerecht werden können (vgl. Mahler et al, 2012).

Das bedeutet, dass alle an der Lehre beteiligten Personengruppen, unabhängig vom Ort der Lehre, die zu lehrenden beruflichen Kompetenzen ,auf die Bewältigung aktueller beruflicher Anforderungen sowie auf die antizipierende Vorbereitung auf zukünftige Herausforderungen“" (von der Heyden, 2013) ausrichten müssen. Dies erfordert ein kontinuierliches, intensives Auseinandersetzen mit der Ausbildung selbst, um die Studierenden sowohl auf die momentanen als auch auf die noch unbekannten, zukünftigen Aufgaben in der Arbeitswelt gut vorzubereiten (vgl. Mahler et al, 2012).

Durch verpflichtende Berufspraktika, welche einen "ausbildungsrelevanten Teil des Studiums darstellen“ (FHStG 2015 \& 3, Abs. 2 Z 3) und welche die Berufsbefähigung sicherstellen sollen (vgl. Lange, 2013), sind auch Ergotherapeuten/-innen aus dem hochschulexternen Umfeld in die Lehre involviert. Mit der Entscheidung, in der Praktikumsanleitung tätig zu sein, übernimmt diese Person die Verantwortung über die im jeweiligen Curriculum festgehaltenen zu erwerbenden Lehrinhalte und Kompetenzen. Führt sie diese Funktion aus, ist sie in dieser Hinsicht Lehrenden an Fachhochschulen gleichgestellt und kann somit als „Praxislehrende/-r" bezeichnet werden.

Die meisten medizinischen Einrichtungen Österreichs stellen derzeit ergotherapeutische Praktikumsstellen zur Verfügung, in denen die erforderliche Personalund Sachausstattung sowie die Durchführung der therapeutischen oder diagnostischen Maßnahmen und Verfahren gemäß FH-MTD-AV § 3, Abs. 3 sichergestellt sind. Entsprechend dieser Verordnung muss auch „die Vermittlung der erforderlichen Kenntnisse, Fähigkeiten und Fertigkeiten gewährleistet" (ebenda) sein. Um diesem Auftrag nachkommen zu können, bedarf es geeigneter Lehrender an den Praktikumsstellen (vgl. CAOT \& ACOTUP, 2012; AOTA, 2009). Die Qualifikation ergotherapeutischer Praxislehrender ist durch deren Berufserfahrung und einer nicht näher definierten pädagogischen Eignung (vgl. FH-MTD-AV § 6) gegeben.

\section{Relevanz des Themas}

Somit stellte sich die Frage, welche über die fachbereichsspezifische Qualifikation hinausgehenden Fertigkeiten und Fähigkeiten eine Ergotherapeutin oder ein Ergotherapeut für eine hochschulgerechte Praktikumsanleitung benötigt. Der Mangel an Richtlinien oder Orientierungshilfen für aktive Praxislehrende im gesamten MTD-Bereich, welche diesen aufzeigen, ob die eigenen Kompetenzen für die Ausübung einer Praktikumsanleitung ausreichend sind, birgt die Gefahr der unbewussten Inkompetenz (vgl. Linker, 2009). Darüber hinaus wird die Identitätsfindung in dieser Funktion sowie die gesellschaftliche Anerkennung dieser sozialen Position erschwert (vgl. Preyer, 2012). Wer so komplexe Aufgaben zu bewältigen hat, kann die für die Hochschullehre relevante Prozess-, Ergebnis- oder Strukturqualität (vgl. Fink, 2014) nur erfüllen, wenn deren Inhalte und Ziele den Verantwortlichen bewusst sind.

Um in der Situation der Praktikumsanleitung ein Rollenbewusstsein zu entwickeln, bedarf es eines Rollenbildes in Form einer "Collage von Verhaltenserwartungen“ (Preyer, 2012), welche von allen damit tangierten Personengruppen gestaltet wird. Diese werden für Strobel und Westhoff in einem Anforderungsprofildeutlich, dessen Aufgabeim Aufzeigen von differenzierten, zur „Bewältigung der zukünftigen Arbeitsaufgaben notwendigen Anforderungen mit den dazugehörigen Verhaltensweisen“ (2010) liegt.

Mit der Erstellung eines möglichst umfassenden Anforderungsprofils für ergotherapeutische Praxislehrende im Rahmen einer qualitativen Studie sollte eine Grundlage geschaffen werden, die den Aufgabenbereich Praxislehrender sichtbar macht und somit eine vertiefende Betrachtung bzw. Weiterentwicklung ermöglicht.

Um die Anforderungen verschiedenster Interessensgruppen für diese besondere Tätigkeit herauszufinden, wurde folgender grundlegender Forschungsfrage nachgegangen: „Welche Anforderungen werden an die ergotherapeutische Praktikumsanleitung gestellt?"

Ziel der Forschungsarbeit ist es, allen an einer gelungenen ergotherapeutischen Ausbildung interessierten Stakeholdern ein umfassendes Anforderungsprofil für die Ausübung der Praktikumsanleitung zur Verfügung zu stellen. Anhand diesem besteht die Möglichkeit, Wissensbzw. Entwicklungsdefizite in der Praktikumsanleitung zu evaluieren, mit der Absicht, durch unterstützende und 
effektive Weiterbildungsmaßnahmen das Erreichen eines hohen Qualitätsstandards der Berufspraktika im Rahmen der Hochschullehre (vgl. Pfäffli, 2015) sicherzustellen.

\section{METHODE}

\section{Studiendesign}

In der qualitativen empirischen Wissenschaft werden Fokusgruppen vermehrt als Forschungsinstrument zur Informationssammlung $\mathrm{zu}$ einem spezifischen Thema (vgl. Lamnek \& Krell, 2016) eingesetzt. Aus Mangel an einschlägiger Literatur und der hohen Komplexität der Thematik wurde zur Erhebung der für die Ausübung der Praktikumsanleitung benötigten Fertigkeiten und Fähigkeiten diese Form der Gruppendiskussion gewählt. Bei dieser gelingt es, durch die unterschiedlichen, begründeten Sichtweisen der Teilnehmer eine Vielfalt an neuen, zuvor unbedachten Aspekten zu generieren (vgl. Schoch, 2016). Aufgrund der Interaktionen innerhalb der Gruppe wird eine facettenreichere Informationsdichte und -tiefe als bei Einzelinterviews oder schriftlichen Befragungen (vgl. Schulz, 2012) erwartet. Für die Befragung wurde ein induktiver Ansatz gewählt und vorab wurden keine Kategorien postuliert. Diese wurden erst anhand der getroffenen Aussagen und Themen entwickelt.

\section{Einschlusskriterien}

Die Autorinnen entschlossen sich, die Expertise von Praxislehrenden zu nutzen, da diese die tatsächlichen Ansprüche unter Berücksichtigung der unterschiedlichen Rahmenbedingungen kennen und mit ihrem Wissen darüber die externe Validität des Anforderungsprofils erhöhen.

Vor der Datenerhebung wurden relevante Merkmale im Sinne eines Stichprobenplans für die Expertenauswahl definiert, um eine Bandbreite soziostruktureller Einflüsse zu erfassen. Die maximale Größe der Fokusgruppen wurde mit je zehn Personen festgelegt. Es wurden Ergotherapeutinnen und Ergotherapeuten gesucht, welche

- in der Praktikumsanleitung unterschiedlich lange tätig sind. Diese Personengruppe ist unmittelbar mit den Anforderungen konfrontiert und in jeder Hinsicht involviert. Somit verfügt sie über ein ähnliches Vorverständnis bezüglich der Thematik. Die divergente Erfahrung mit Studierenden im Rahmen einer Praktikumsanleitung wurde bewusst gewählt, um eine stärkere Gesprächsdynamik zu erhalten.

- in unterschiedlichen Institutionen und Fachbereichen tätig sind. Ziel dieser Heterogenität ist die möglichst reale Abbildung der ergotherapeutischen Arbeitswelt, in welcher Studierende ihre Berufspraktika absolvieren.
- früher in der Praktikumsanleitung tätig waren und nun an einer Fachhochschule angestellt sind und aus dieser Position die Berufspraktika betreuen. Diese Personengruppe ist mit der Auswahl von geeigneten Praktikumsplätzen betraut. Aufgrund der Rückmeldungen der Studierenden bzw. der Praxislehrenden erhalten sie Einblick sowohl in die erfüllten als auch in die unerfüllten Anforderungen an die Praktikumsanleitung.

\section{Rekrutierung}

Nach einer kurzen mündlichen Präsentation des Forschungsvorhabens auf der Fachtagung des Verbandes österreichischer Ergotherapeutinnen und Ergotherapeuten sowie am Praktikumsanleitungstag 2017 der FH Campus Wien meldeten sich 14 Interessierte bei derErstautorin. Die Teilnehmenden wurden in einer Face-to-face-Einladung über die Eckdaten der Fokusgruppen informiert. Im Rahmen des zu unterzeichnenden „Informed Consent“ wurden sie entsprechend der Helsinki-Deklaration (WMA, 2013) über ethische Aspekte wie die freiwillige Teilnahme, Zweck der Studie, Ablauf der Fokusgruppe, Anonymisierung der Daten und Aufwandsentschädigung aufgeklärt. Eingeschlossen wurden alle, da sie den Anforderungen des Stichprobenplans entsprachen und eine ausreichende Diversität hinsichtlich Berufserfahrung, Alter, Arbeitsfeld, Arbeits-Setting und Arbeitsort gegeben war.

\section{Datenerhebung}

Ende April und Anfang Mai wurden im Rahmen von zwei Fokusgruppen zu je 2,5 Stunden mit der Leitfrage „Welche Anforderungen werden an die Praktikumsanleitung gestellt?“ detaillierte Inhalte erarbeitet. Eine Fokusgruppe fand in Wien, die andere in Linz statt, um eventuell auftretende regionale Unterschiede miteinzubeziehen und die Anfahrtswege gering $\mathrm{zu}$ halten. Externe Moderatorinnen, welche sowohl im ergotherapeutischen Berufsfeld als auch in der Moderation langjährige Erfahrung besitzen, führten durch den zuvor festgelegten Ablauf, um die Vergleichbarkeit der beiden Gruppen zu ermöglichen. Ihr Auftrag war neben der Verfolgung der Fragestellung mit einer neutralen Einstellung das Festhalten des Gesagten und das Stellen von Verständnisfragen im Sinne eines member checkings, um inhaltliche Nuancen zu differenzieren und zu klären. Unter Verwendung einer Videokamera wurden die Aussagen aufgezeichnet, die Erstautorin war anwesend und hielt die Inhalte in Form schriftlicher Notizen fest.

Die Teilnehmenden formulierten entsprechend der vorgegebenen Abfolge in Kleingruppen Anforderungen, 


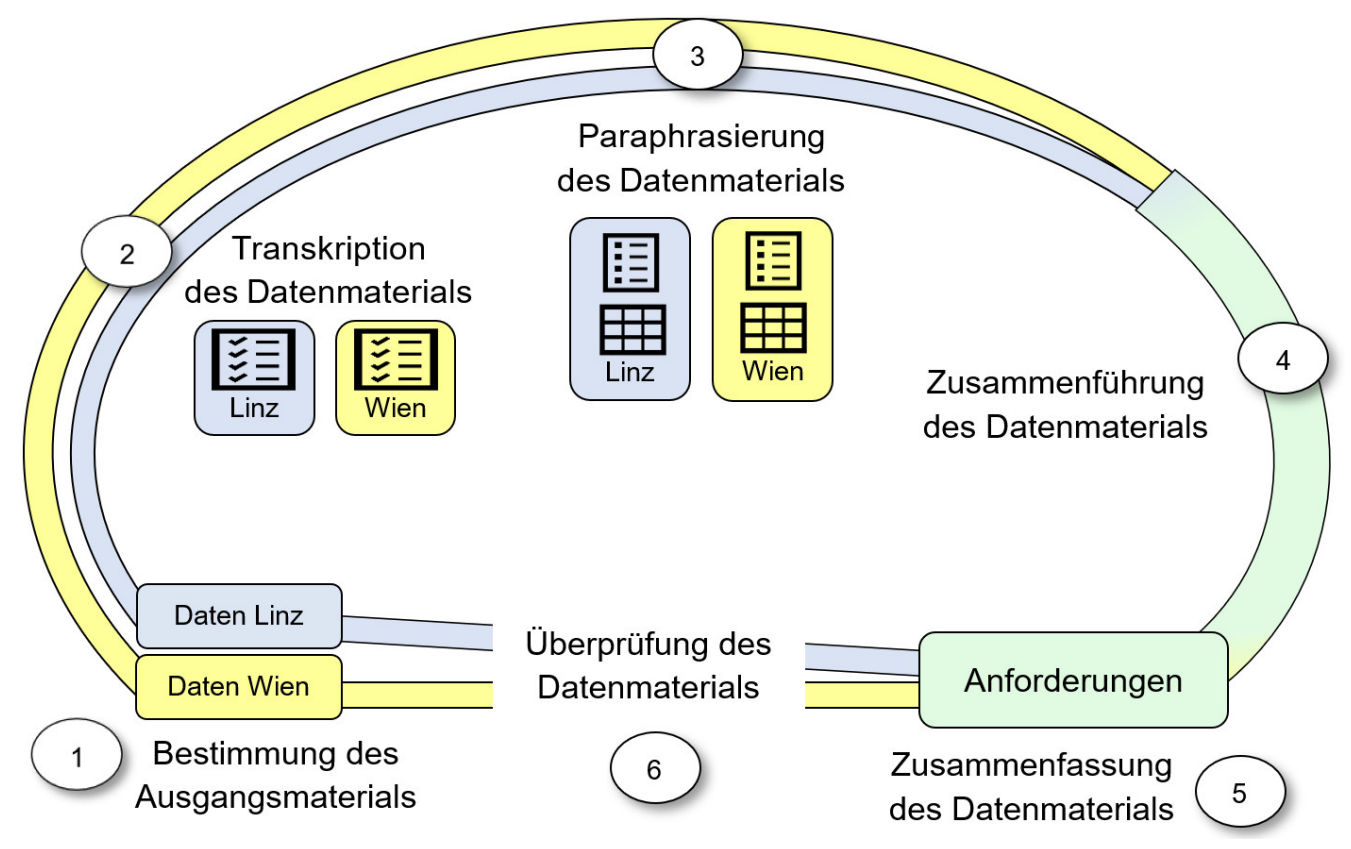

Abbildung 1: Prozess der qualitativen Inhaltsanalyse.

welche anschließend im Plenum diskutiert, weiter ausgeführt, auf einer Pinnwand geclustert und strukturiert wurden. Noch während der Fokusgruppen erfolgte eine erste Kategorisierung der Ergebnisse durch die Untersuchungsteilnehmenden. Es wurden - basierend auf den behandelten Themen - induktiv erste Kategorien gebildet.

Die erste Fokusgruppe wurde als Pilot ausgerichtet. Da dieser erfolgreich verlief, waren keine Änderungen im Ablauf notwendig. Die zweite Fokusgruppe konnte daraufhin im gleichen Modus durchgeführt werden. Die gewonnenen Daten waren vergleichbar und konnten zusammengeführt werden.

\section{Datenanalyse und Datenmanagement}

Die erhobenen Daten wurden anonymisiert transkribiert, die Transkripte codiert. Dieses Datenmaterial wurde von beiden Autorinnen gemäß den Kriterien einer Triangulation (vgl. Flick, 2011) unabhängig voneinander mithilfe einer zusammenfassenden qualitativen Inhaltsanalyse nach Mayring (vgl. 2015) zu einem Anforderungsprofil synthetisiert, wobei die in Abbildung 1 visualisierten Schritte (3)-(5) in Etappen bis zu deren Übereinstimmung gegenübergestellt wurden. Der Forschungsgegenstand konnte dabei intensiv analysiert und ein Bezug zwischen den individuellen Aussagen zu disziplinären Haltungen und Sichtweisen erstellt werden.

(1) Als Erstes wurde das Ausgangsmaterial bestimmt und eine

(2) Transkription des Datenmaterials durchgeführt.
(3) Danach wurden die Aussagen durch Paraphrasierung so weit modifiziert, dass ,nicht inhaltstragende Textbestandteile“ (Mayring, 2015) entfernt wurden und unter Beibehaltung des originalen Standpunktes ein vergleichbares Sprachniveau hergestellt wurde. Aus beiden Fokusgruppen wurde jeweils eine Teilnehmende mit den paraphrasierten Daten konfrontiert und um Feedback bezüglich korrekter Interpretation gebeten.

(4) Anschließend kam es zur Zusammenführung des Datenmaterials, wobei die Aussagen analysiert und kategorisiert wurden.

(5) In einem nächsten Schritt wurde in Form einer Zusammenfassung das Material durch den induktiven Prozess der Abstraktion so weit reduziert, dass der übriggebliebene Inhalt noch immer das Abbild des ursprünglichen Grundmaterials darstellt.

(6) Zuletzt wurde jeder einzelne Inhalt aus dem Ausgangsmaterial beider Fokusgruppen Kategorien zugeordnet und auf dessen Sichtbarwerden im Endmaterial überprüft.

Die Daten werden verschlüsselt in elektronischer Form von der Erstautorin ,zur Sicherung guter wissenschaftlicher Praxis“ (DFG, 2013) sicher verwahrt und nach einer Aufbewahrungszeit von zehn Jahren gelöscht. Zur Gewährleistung der Vollständigkeit der Berichterstattung wurden die Consolidated criteria for reporting qualitative research (COREQ; Tong, Sainsbury, \& Craig, 2007) angewendet. 


\section{ERGEBNISSE}

\section{Informationen zur Stichprobe}

Insgesamt nahmen 14 Praxislehrende, 6 am Standort Wien und 8 am Standort Linz, teil und brachten eine hohe Bereitschaft zur aktiven Beteiligung mit. Sie waren zwischen 31 und 56 Jahre alt, das Durchschnittsalter lag bei 44,9 Jahren. Eine Diversität bezüglich Berufserfahrung ist durch die Spannweite von 8-31 Jahren gegeben. In Hinblick auf Erfahrung in der Praktikumsanleitung liegt die Streuung zwischen 3 und 25 Jahren. Die Teilnehmenden üben ihre Tätigkeit im angestellten, freiberuflichen und/oder lehrenden Bereich aus. Die beiden Männer und 12 Frauen haben ihre Arbeitsstellen in Wien, Niederösterreich, Oberösterreich und der Steiermark und sind in den Fachbereichen Neurologie, Orthopädie, Geriatrie, Pädiatrie und Psychiatrie tätig.

\section{Anforderungen an Praxislehrende}

In beiden Fokusgruppen erfassten die Praxislehrenden im Kollektiv und mit einer theoretischen Sättigung von 92,68 \% ein großes Ausmaß an Anforderungen. Diese ergeben sich einerseits in der direkten Interaktion mit Studierenden, andererseits aus der persönlichen Rolle der Praxislehrenden. Der Großteil der Anforderungen entstammt, wie in Tabelle 1 grün hinterlegt, der personalen Dimension. Der blau hinterlegte, fachlichmethodische Anteil erhält in der direkten Arbeit mit Studierenden Relevanz.

Folgende Anforderungen konnten identifiziert werden (vgl. Tabelle 1).

\section{Anforderungen in der direkten Interaktion mit Studierenden aus dem fachlich-methodischen Bereich}

Aus Sicht der Praxislehrenden ist eine zu Beginn des Berufspraktikums erfolgende Erfassung der Rahmenbedingungen, wie in Anforderung 1 angeführt, wichtig „,... im Sinne von Transparenz in der organisatorischen Struktur des Praktikums" (TN_ b210_16). Die fachliche Durchführbarkeit impliziert das Kennen des Ausgangspunktes, den Ausbildungsstand der Studierenden als auch des Endpunktes, die bis dahin zu erwerbenden Kompetenzen und Inhalte sowie die Notwendigkeit, „(...) auch Erwartungen abzustecken“ (TN_b210_58). Als eine große Anforderung wird empfunden, alle Vorgaben, sowohl die innerbetrieblichen als auch die der Fachhochschulen bzw. der Lehre in Einklang zu bringen. „Ich brauch ein gewisses Organisationstalent (...) oder auch Zeitmanagement" (TN_f107_30).
Eine weitere Anforderung ist das Schaffen von Lernsituationen (vgl. Anforderung 2). Dies geht einher mit der Modifikation der Lernziele mit ihren Dimensionen und Stufen im jeweiligen Kontext, ,(..) abgestimmt auf den Studenten“ (TN_f107_58). Die Praxislehrenden sehen sich gefordert, zu „schauen, bei welchem Patienten kann der Praktikant das und das üben“ (TN_a200_01). Somit müssen sie über Kenntnis der verschiedenartigen Lernzielstufen und Dimensionen verfügen, um den aktuellen Entwicklungsstand der bzw. des Studierenden feststellen zu können. „Ich muss immer schauen, wo stehen die Praktikanten? (...) Wie ist der aktuelle Wissensstand?" (TN_b117_15). Erst dann sind das gezielte Gestalten der Lernumgebung bzw. die Auswahl der Patienten möglich.

Unter der Begrifflichkeit der Anleitung wird eine eigene Didaktik beschrieben (vgl. Anforderung 3). Auch wenn davon ausgegangen wird, „(..) man kann das als Ergotherapeutin, man kann sich auf das Gegenüber einstellen, es ist trotzdem etwas anderes, $o b$ das eine Kollegin in Ausbildung oder ein Patient ist“ (TN_m116_49). Diese Form der Didaktik ,(..) ist ganz nahe an Aufgaben stellen, leiten, führen. Da steht Einfühlungsvermögen und Flexibilität darüber" (TN_p322_24). Beides wird von den Praxislehrenden als notwendig erachtet, „(...) um zu schauen: wo steht die Praktikantin, was bringt sie schon mit (...)“ $\left(\mathrm{TN}_{-}\right.$ p322_24). Diese individuellen Faktoren Studierender beziehen sich auf die fachlichen Ebenen und auf den persönlichen Lerntyp. Das Anleiten, Begleiten und Supervidieren wird als Herausforderung betrachtet, „(...) dass wenn ich A sage, dass der A versteht" $\left(\mathrm{TN}_{-}\right.$ b124_50), aber auch in Bezug auf ,(...) wie viel sage ich, wie viel sage ich nicht?" (TN_m127_08).

Die Umwandlung von implizitem zu explizitem Wissen wird als Herausforderung betrachtet (vgl. Anforderung 4). „Wir haben schon alle so einen Wissens-Pool, man verknüpft ja schon so viel im Gehirn, wo der Student überhaupt keine Ahnung hat, und dann muss man schon verschiedene Bereiche erläutern, dass der checkt, wie komme ich jetzt eigentlich zu dem Schluss. " (TN_ o125_03). Über den Prozess der Selbstreflexion werden sich die Praxislehrenden der Beweggründe ihrer intuitiven Handlungen bewusst, sodass diese durch die Verwendung einer passenden Wortwahl von den Studierenden analytisch nachvollzogen werden können. „Man arbeitet mit zunehmendem Berufsalter intuitiv, (...) bei Begleitung einer Studentin muss man in einer geordneten Weise das eigene Tun Schritt für Schritt aussprechen." (TN_ k324_10). Hier sehen sich die Praxislehrenden in einer wichtigen Rolle, durch das „(..) Transparentmachen gewisser Prozesse, die einem während der Arbeit am Patienten begleiten“ (TN_b117_41) aus reflektierter Erfahrung mitteilbares Wissen zu generieren. 


\section{Anforderungen an ergotherapeutische Praxislehrende}

1) Sie organisieren eine für alle Beteiligten transparente Praktikumsstruktur entsprechend den spezifischen Anforderungen der Ausbildungsstätte der/des Studierenden und gewährleisten die zeitliche und fachliche Durchführbarkeit innerhalb des betrieblichen Rahmens.

2) Sie schaffen Lernsituationen, in welchen die Studierenden ihre Kompetenzen in Ausrichtung auf die beabsichtigten Lernergebnisse anwenden, erweitern und vertiefen können.

3) Sie leiten an, begleiten bzw. supervidieren unter Berücksichtigung des Lernniveaus und Lerntypus der Studierenden den ergotherapeutischen Prozess mit dem Ziel der Handlungsbefähigung.

4) Sie reflektieren ihre impliziten Annahmen während und nach einer Handlung, um diese für Studierende nachvollziehbar zu machen

5) Sie begleiten mit konstruktivem Feedback Lern- und Entwicklungsprozesse der Studierenden formativ.

6) Sie beurteilen anhand transparenter Beurteilungskriterien objektiv und nachvollziehbar den an den Lernzielen ausgerichteten Kompetenzzuwachs der Studierenden.

7) Sie setzen sich mit evidenz-basiertem Arbeiten auseinander und reflektieren mit den Studierenden ihre therapeutischen Entscheidungen hinsichtlich wissenschaftlicher Erkenntnisse.

8) Sie analysieren Teamentwicklungsprozesse und setzen bei Bedarf Interventionen, um den Lernprozess zu ermöglichen.

9) Sie unterstützen die Studierenden in belastenden Situationen in ihrer Persönlichkeitsentwicklung

10) Sie verfügen über eine positive Berufsidentität, sind sich ihrer Rolle und der damit verbundenen Aufgaben bewusst und besitzen das notwendige Engagement zur Ausführung dieser.

11) Sie nehmen über authentisches, wertschätzendes und empathisches Agieren eine professionelle Haltung ein.

12) Sie repräsentieren gegenüber den Studierenden die eigene Arbeitsstelle sowie den eigenen Fachbereich, gegenüber den Patientinnen und Patienten die Selbstverständlichkeit, eine Ausbildungsstelle zu sein.

13) Sie stellen sowohl Kommunikation als auch soziale Beziehungen bewusst, konstruktiv und zielorientiert her und gestalten diese.

14) Sie fühlen sich in ihrem Fachbereich sicher und sind bereit, ihr fachliches Wissen und ihre Erfahrungen an die zukünftigen Berufskolleginnen und -kollegen weiterzugeben.

15) Sie übernehmen das an die Rolle gebundene Mehr an Verantwortung.

16) Sie sind persönlich stabil, nehmen ihre Bedürfnisse wahr und setzen respektvoll Grenzen.

17) Sie reflektieren ihr Denken, Handeln und ihre Erfahrungen, sind zur fachlichen und persönlichen Weiterentwicklung bereit und integrieren Neues im Sinne von lebenslangem Lernen.

18) Sie besitzen Flexibilität, Ausdauer und die Gelassenheit, schwierigen Situationen mit Erfahrungswissen und Humor zu begegnen.

Tabelle 1: Übersicht der Anforderungen.

„Man muss Feedback geben als Anleiter“(TN_b113_43), „verbal, aber auch nonverbal“ (TN_f114_01), „und das möglichst objektiv und konstruktiv, sodass der Student im besten Fall etwas lernen kann" (TN_f108_57). Begleitendes konstruktives Feedback orientiert sich an der Situation, ist demnach bedarfsorientiert und ,(...) hat Entwicklung in sich“(TN_x327_43). Feedback bringt das Potenzial der Einnahme eines Perspektivenwechsels mit und wird von den Praxislehrenden, wie in Anforderung 5 beschrieben, auch eingesetzt, ,....) um Studierende beim Wissenstransfer Theorie - Praxis, Praxis - Theorie zu unterstützen“(TN_g317_53).

Einen wesentlichen Bereich stellt die Beurteilung (vgl. Anforderung 6) dar. „Das ist eine wichtige Anforderung, Herausforderung, der man sich als PA stellen muss. Auch geübt werden, objektiv Noten zu geben“"(TN_b112_42). In beiden Fokusgruppen zeigte sich die Beurteilung als eine unliebsame Anforderung, die elementare Bedeutung der Erfassung der beruflichen Handlungskompetenz ist dabei gegeben. Die Praxislehrenden empfinden den Rollenwechsel vom Betreuenden zum Beurteilenden als große Herausforderung. Mangelnde Kenntnisse bezüglich Anwendung von Beurteilungskriterien, welche eine transparente Beurteilungsform mit sich bringen, tragen zusätzlich zur Verunsicherung bei.

Das Wissen, „(...) es ist Evidence Based Practice gefordert“" (TN_b110_46), ist an den Praktikumsstellen vorhanden. Wie in Anforderung 7 beschrieben, wird es von den Experten/-innen in der Praxislehre als notwendig erachtet, sich mit diesem Themenbereich auseinanderzusetzen und „(...) die Bereitschaft, sich auf etwas Neues einzulassen, was ich vielleicht noch nicht weiß, und da auch mitzulernen mit denen (Anm.: den Studierenden)“ (TN_s113_07), mitzubringen. Sie sehen „(..) in der Kombination mit der FH die theoretische Auseinandersetzung“ (TN_m111_38) mit evidenzbasiertem Wissen. Durch die Integration dieses Wissens in die berufliche Praxis über Reflexion mit den 
Studierenden ist es ihnen möglich, „(...) am Puls der Zeit“(TN_x319_33) zu bleiben.

\section{Anforderungen in der direkten Interaktion mit Studierenden aus dem personalen Bereich}

Die Entscheidung, „(..) nehmen wir Praktikanten mit (...)“ (TN_n312_56), hat meistens ebenso auf das ergotherapeutische Team der Institution Auswirkungen. „Es braucht eine Vertretung, es braucht Regelungen, wenn wer krank ist, wenn wer im Urlaub ist" (TN_ b114_34). Studierende zu betreuen, betrifft ,(...) nicht nur mein persönliches Zeitmanagement, sondern auch das vom Team, es sind ja meist mehr Leute involviert" (TN_m115_01). Die Praxislehrenden sehen hier ihre Aufgabe im offenen Ansprechen dieser Tatsache, damit Erwartungen besprochen, Rollen und Aufgaben neu verteilt werden können (vgl. Anforderung 8).

Die Möglichkeiten, im Berufspraktikum an seine Grenzen zu gelangen, sind besonders bei jüngeren Studierenden sehr vielfältig. , Wenn sie merken, es ist so viel (...)“(TN_ m203_49), ,oder wenn Praktikanten persönlich betroffen sind, wie z. B. mein Papa ist auch Alkoholiker oder mein Onkel hat ein Schädel-Hirn-Trauma“ (TN_m203_28), sehen sich die Experten/-innen als Praxislehrende gefordert (vgl. Anforderung 9). „Da macht man ganz viel supervisorische Arbeit (...) und du machst dann natürlich dieses Mehr an Verantwortung, du hast sie ja auch für dein Anhängsel, aber gleichzeitig ist es auch wichtig, dass man ihnen das bewusst macht"(TN_m204_07).

\section{Anforderungen in der persönlichen Rolle derl des Praxislehrenden}

Die positive Berufsidentität spiegelt sich in der Haltung der jeweiligen Person, in diesem Fall der Praxislehrenden, wider im Sinne von „Ich bin gerne Ergotherapeutin und vermittle das gerne, was ich tue“ (TN_p311_25). Bezüglich Rollenbewusstsein äußersten sich die Teilnehmer/innen so: „Derzeit ist eine Eigendefinition notwendig, es gibt keine fertige Vorstellung, was ein Praktikumsanleiter alles tun und können soll. Es gibt kein ,so sollst du sein'. So hat jede Praktikumsstelle ihre Rolle definiert (...)“(TN_x312_28).

Die Praxislehrenden sollten sich bezüglich der eigenen Aufgaben, der Aufgaben der Studierenden und der Fachhochschule im Klaren sein (vgl. Anforderung 10). Nachdem die Betreuung Studierender nicht anstelle des ergotherapeutischen Berufsalltags, sondern als Zusatzfunktion in diesen integriert werden muss, wird diese Aufgabe immer mit einem Mehraufwand gesehen: „Ich muss motiviert sein, dass ich die Zeit so komprimiere, dass sich das (Anm.: die Anleitung) ausgeht" (TN x315_31). Keinem der Fokusgruppenteilnehmenden wird Zeit für die Praktikumsanleitung zur Verfügung gestellt.
Je geringer die Passung zwischen Praktikumslehrenden und Studierenden ist, umso mehr bedarf es einer professionellen Haltung, denn „Manchmal passt die Chemie, aber das ist ja nicht immer so" (TN_x317_07) und des Bewusstseins „Nicht, dass ich dann den Praktikanten das spüren lasse oder an ihm auslasse" (TN_ o218_24). Diese Anforderung wird in enger Verbindung zur persönlichen Stabilität gesehen, „(...) in dem Sinn, dass ich meine eigene persönliche Befindlichkeit hintanstelle (...)“ (TN_e315_56). In Anforderung 11 wird als wichtig erachtet, seine eigenen Impulse zu erkennen, unterzuordnen und mit einer professionellen Haltung herausfordernde Situationen zu meistern.

Die Tätigkeit der Praktikumsanleitung wird von den Experten/-innen auch „(...) ein Stück weit als Öffentlichkeitsarbeit““ (TN_v209_17) gesehen (vgl. Anforderung 12). Den Studierenden werden in der Regel die Institutionen mit ihrem Leitbild bzw. ihrer Philosophie vorgestellt, welche wiederum ihre Eindrücke und Erfahrungen aus dem Praktikum an die Außenwelt überliefern. Als weitere Anforderung wird die Präsentation des Arbeitsplatzes als „Ausbildungsstelle der Fachhochschulen“ (TN_s222_39) gegenüber den Patientinnen und Patienten wahrgenommen. „Das ist die zukünftige Kollegin und die bringt die neuesten wissenschaftlichen Erkenntnisse mit. Es ist immer eine Sache der Vermittlung" (TN_s224_43).

Praxislehrende stehen im Informationsaustausch „(..) mit der Fachhochschule, mit Studierenden, auch mit dem Team oder Patienten und Angehörigen. (...) Kommunikationsfähigkeit ist natürlich immer die Grundlage für alles, ich kommuniziere auf sehr vielen verschiedenen Ebenen als Anleiter" (TN_f105_22). Weiters erwähnt wurden noch Vorgesetzte und der Dienstgeber, um Informationen aller Art auszutauschen. In Situationen, in welchen dritte Personen, wie Patientinnen oder Patienten, involviert sind, ist nicht nur verbales Geschick notwendig, „(...) es lauft ja nicht immer alles konfliktfrei ab"(TN_c318_07), sondern auch das Gespür, wer wann wie viel Aufmerksamkeit benötigt, um den Lern- bzw. Therapiezielen näher zu kommen (vgl. Anforderung 13).

Die Forderung nach facheinschlägiger Berufserfahrung wird in Anforderung 14 bestätigt, denn „Berufserfahrung hat sehr viel mit Sicherheit zu tun, (...) wie sicher fühle ich mich als Anleiterin in meiner Fachkompetenz?" (TN_f104_31) und ,(..) fühle ich mich auch sicher genug, das weiterzugeben?" (TN_m212_28). In einer der beiden Fokusgruppen wurde die Anzahl an Jahren, die man braucht, um erfahren genug zu sein, diskutiert, mit dem Ergebnis, dass „(...) das sehr individuell betrachtet werden muss und nur jede bzw. jeder für sich selbst entscheiden kann" (TN_104_50).

Das Bewusstsein „Ich habe eine Verantwortung für die Praktikanten in Richtung Lernprozess, in Richtung 
Anleitung, es ist einfach ein Mehr an Verantwortung" (TN_b115_09) ist bei allen Fokusgruppenteilnehmenden gegeben und wird in folgender Aussage präzisiert: „Man sollte schon das Bild der Verantwortung haben: man bildet Kollegen aus" (TN_x435_31) und schließt für die Experten/-innen die fachliche Aktualität ihres ergotherapeutischen Handelns mit ein (vgl. Anforderung 15).

Persönliche Stabilität, wie in Anforderung 16 beschrieben, bedeutet in diesem Kontext, „(...), dass es günstig ist, total in sich zu ruhen, dann kann ich auch sagen, ich will heute lieber alleine Mittagessen" (TN_ p506_38). Hier kommt den Praxislehrenden eine enorme Vorbildfunktion zu im Sinne von wertschätzendem Verbalisieren eigener Bedarfe, im Setzen von Grenzen und im Ermöglichen eines Minimums an Privatsphäre, denn „Wir sind den ganzen Tag beisammen - sie sind acht Stunden am Tag da, sitzen beim Essen neben einem, fahren vielleicht mit dem gleichen Zug“ (TN_b213_42). Darüber hinaus gilt es stets abzuwägen: „Bis wie weit oder wo sind sie jetzt dabei? Das betrifft einerseits das Team andererseits die Arbeit mit Klienten“"(TN_c503_28). Es entstehen regelmäßig widersprüchliche und konflikthafte Anforderungen, welche die Frage aufwerfen: „Wie gehe ich selbst damit um?" (TN_o214_07).

„Wie wirken die Fragen der Studierenden auf mich, welche Emotionen lösen sie in mir selbst aus und wie reagiere ich darauf?" (TN_f105_28). Die Fähigkeit, das Erlebende bzw. Erlebte kognitiv zu betrachten und selbstkritisch zu reflektieren, wird von den Praxislehrenden in dieser Rolle als wichtig erachtet (vgl. Anforderung 17). In der Aussage „Da hilft reflektierte Erfahrung - und da hilft ein Gegenüber sehr, oder auch ein Team“(TN_g514_56) wird die Anforderung, sich selbst Feedback im Austausch mit Teamkolleginnen und -kollegen zu holen, sichtbar. „Durch das, was vom Student kommt, passiert bei mir (...) Weiterentwicklung" (TN_m122_53) und ermöglicht fortlaufendes Lernen im professionellen Sinne. Der reflexive Umgang mit derartigen Situationen durch die Praxislehrenden wird von diesen als wertvoller Prozess in der Vertiefung der eigenen Kompetenzen betrachtet. Die Erkenntnis, „Ich muss einfach die Fähigkeit haben, an der Aufgabe zu wachsen, meine Erfahrungen zu reflektieren und einzubauen, und mir, wenn ich dann selber merke, mir fehlt das, dass ich mir das aneigne, ganz bewusst“"(TN_e515_29), entspricht der Forderung nach Weiterbildung und Weiterentwicklung im Sinne von lebenslangem Lernen.

Die Anforderung 18, flexibel zu sein, wird von beiden Fokusgruppen stark wahrgenommen. Flexibilität betreffend „(...) unterschiedliche Personentypen, unterschiedliche Semester, Ausbildungsinhalte (...)“ (TN_p508_43) verlangt eine Vielfalt von Variationsmöglichkeiten, um Lernumgebungen für die Studierenden zu schaffen und somit deren Kompetenzerwerb zu ermöglichen. Hier erweist sich die „Gelassenheit als wichtige Kompetenz neben der Ausdauer und dem Engagement" (TN_x318_44). Der Schatz des Erfahrungswissens wird als wertvolle Ressource gesehen und auch der Sinn für Humor hilft, „(...) nicht alles ganz so ernst zu nehmen, eine gewisse Stimmung“" (TN_c318_56) zu verbreiten, um längere Zeit in der Praktikumsanleitung tätig zu sein.

\section{DISKUSSION}

Die Ergebnisse der beiden Fokusgruppen zeigen, dass sich die Teilnehmenden während der Begleitung Studierender im Berufspraktikum mit 18 differenzierten Anforderungen konfrontiert sehen. Um diesen gerecht zu werden, sind für ergotherapeutische Praxislehrende neben ausreichender Berufserfahrung nicht nur überfachliche Kompetenzen, sondern zusätzlich eine didaktische Fachkompetenz zur Entwicklung einer für die Praxislehrenden notwendigen Handlungskompetenz erforderlich (vgl. Kellner-Fichtl, 2016; AOTA, 2009). Diese bietet die Grundlage für ein gelungenes Berufspraktikum, bei welchem nicht nur Handlungskompetenzen in Form von Fach-, Methoden-, Sozial- und Selbstkompetenzen (vgl. Grunert, 2012) erweitert werden sollen, sondern auch die Bildung und Überprüfung einer beruflichen Handlungskompetenz ermöglicht wird (vgl. Brinker, 2009).

Anforderung 1 beschreibt die Wichtigkeit, die für das Berufspraktikum relevanten Rahmenbedingungen $\mathrm{zu}$ identifizieren und diese sich selbst und den Studierenden zunutze zu machen (vgl. Wright, 2009). Die fachliche Ausrichtung impliziert das Kennen des Ausgangspunktes, den Ausbildungsstand der Studierenden, als auch des Endpunktes, die bis dahin zu erwerbenden Kompetenzen und Inhalte. Die Orientierung an den FH-spezifischen Anforderungen beinhaltet die Kenntnisse dieser. Als eine große Anforderung wird empfunden, alle Vorgaben, sowohl die innerbetrieblichen als auch die der Fachhochschulen bzw. der Lehre in Einklang zu bringen. Dazu kommt noch, dass ständig die Lernbedarfe der Studierenden durch die Praktikumsanleitung präzisiert werden müssen (vgl. Wright, 2009). In diesem Zuge wird Praktikumsanleitung mit einem komplexen System verglichen.

Der Terminus „Anleiten“ wird abhängig vom jeweiligen Kontext unterschiedlich aufgefasst. Im Zusammenhang mit Lernen bedeutet es einen systematisierten, ,geplanten und reflektierten Lernprozess" (Ewers, 2001), mit dem Ziel, die berufliche Handlungskompetenz zu erhöhen bzw. zu optimieren (vgl. Mamerow, 2016). Zwischen einer klaren Anleitung zum selbständigen Durchführen unter Supervision liegen noch andere Schritte, wie zu unterstützen, zu begleiten, „durch Impulse zu stimulieren 
oder sogar zu provozieren“ (Webler, 2004). Wie das effizient und damit ressourcenschonend gelingen kann, ist für die in dieser Handlungskompetenz in der Regel meist nicht ausgebildete Praktikumsanleitung eine enorme Herausforderung, welche in Anforderung 3 zum Ausdruck gebracht wird.

Implizites Wissen sichtbar zu machen (vgl. Anforderung 4) ist besonders für die Praktikumsanleitung mit langjähriger Expertise eine große Herausforderung, da diese selbst erst Zugang zum eigenen verborgenen Wissen finden muss (vgl. Martens \& Asbrand, 2009). Im Austausch mit Studierenden bieten sich für beide Seiten wertvolle Gelegenheiten, durch Reflexion das eigene Tun zu beleuchten und mit vorhandenen Konzepten oder Modellen in Beziehung zu bringen.

Anforderung 9 betont die Notwendigkeit, dass PraxislehrendedieStudierendenbeimErwerbindividueller Bewältigungsstrategien durch Unterstützung und die Bereitstellung des erforderlichen Raumes begleiten (vgl. Perkhofer-Czapek \& Potzmann, 2016). Damit wird diesen ermöglicht, ihre bisherigen Erfahrungen mit dem im Rahmen der Profession Ergotherapie momentan Erlebten in Beziehung zu bringen und so ihre Persönlichkeit im Sinne einer „Bildung jenseits der Zweckausbildung“ (Becker, 2009) weiterzuentwickeln.

Lernen im realen Umfeld ist definitiv „,der stärkste Moment in der Generierung von berufspraktischem Wissen“ (Markowitsch 2001, S. 138). Die Bedeutung des Berufspraktikums als Ort der Bildung einer professionellen Haltung wird von den Praxislehrenden durchaus erkannt (siehe Anforderung 10). Deren positive Einstellung zur ergotherapeutischen Tätigkeit bildet die Grundlage zur Entwicklung einer eigenen Berufsidentität (vgl. Seithe, 2012). Das ermöglicht den Studierenden, durch Lernen am Vorbild ihre eigene Rolle als Ergotherapeut/-in zu entwickeln und somit Sicherheit im Tun zu erlangen (vgl. Eberle, 2016). Im Gesundheitsbereich finden sich nur in der Pflege bereits definierte Vorstellungen über die Aufgaben Praxislehrender (vgl. Mamerow, 2016), die die Identifikation mit dieser sozialen Position und somit deren gesellschaftliche Aufwertung unterstützen (vgl. Preyer, 2012).

Mit der Anforderung 13 wird auch Bezug zu den Herausforderungen in schwierigen Situationen genommen, in welchen eine wertschätzende Haltung der Praxislehrenden wesentlich zurerfolgreichen Bewältigung gesehen wird (vgl. Heigl, 2014). Im Lehr-Lern-Prozess sind solche Gegebenheiten durch unterschiedliche Auffassungen zu Vorgehens- oder Verhaltensweisen gekennzeichnet, wobei die Unstimmigkeit zumindest für eine involvierte Person von großer Bedeutung ist und aufgrund des anstehenden Klärungsbedarfs eine starke emotionale Komponente in sich trägt (vgl. Lehmann, 2017). Als Herausforderung wird die Gestaltung der
Praxislehrenden-Studierenden-Beziehung gesehen, da sie den Lern- und Entwicklungsprozess der Studierenden konstruktiv unterstützt und trotz des meist kollegialen Verhältnisses den Rollenwechsel zur lehrenden und beurteilenden Person ermöglicht (vgl. Kellner-Fichtl, 2016).

Die gesetzliche Forderung nach einer „mindestens einjährigen facheinschlägigen Berufserfahrung in einem für das jeweilige Praktikum relevanten Berufsfeld" (FH-MTDAV § 6) wird in Anforderung 14 bestätigt. Als wichtiges Kriterium für eine gelingende Praktikumsanleitung wird die Bereitschaft der Fachexperten/-innen zu lebenslangem Lernen und zur Wissensbereitstellung gesehen (vgl. Anforderung 17). Allerdings unterstützen mangelnde Weiterbildungsmöglichkeiten in diesem Bereich die unreflektierte Anwendung subjektiver Präkonzepte in der Arbeit mit Studierenden (vgl. Krinner, Kruse-Weber, \& Marin, 2016).

Das Ausmaß der von den Experten/-innen formulierten Anforderungen an Praxislehrende umfasst eine wesentlich größere Dimension als die aus den Gesetzen abgeleiteten Mindestanforderungen. Sind hier vor allem Anforderungen aus dem fachlichen, methodischen und didaktisch-pädagogischen Bereich abgebildet (vgl. FHStG $\S 3$, Abs. 2; FHStG $\S 8$, Abs. 3), kommen noch wichtige Anforderungen aus dem sozialen und personalen Bereich dazu. Zu diesem Ergebnis kam auch Kellner-Fichtl (2016) und die Arbeitsgruppe der AOTA. Anforderungen aus dem sozialen Bereich wurden nicht explizit deklariert, da sie in direkter Verbindung mit effizientem Verhalten in sozialen Situationen stehen (vgl. Grunwald \& Steinbacher, 2014). Somit sind sie für Praxislehrende in der erfolgreichen Begegnung aller Anforderungen präsent.

Die Ausbildung in den gehobenen medizinischtechnischen Diensten basiert auf der gleichen Gesetzeslage. Die Anforderungen an Praxislehrende, die über eine fachliche Qualifikation hinausgehen, sind ähnlich. Insofern hat das erstellte Anforderungsprofil auch Relevanz für die übrigen MTD-Berufe. Es ist auch festzustellen, dass es, im Gegensatz zum Pflegebereich, noch keine Literatur bezüglich dieses Themas für diese Professionen gibt.

\section{Diskussion des methodischen Vorgehens}

Fokusgruppen stellen eine geeignete Herangehensweise für die Erforschung komplexer Inhalte dar. Die gewählte Methodik erwies sich als zielführend, um Daten bezüglich der Anforderungen an Praxislehrende zu gewinnen bzw. zu generieren. Durch die sorgfältige Auswahl der Teilnehmenden und durch das Durchführen zweier Fokusgruppen an unterschiedlichen Orten gelang es, ein der Realität nahes Bild der Heterogenität und Vielfalt der 
Praxislehrenden abzubilden. Dies spiegelte sich auch in einem zwar ähnlichen, aber nicht exakt gleichen Verlauf der Gruppenprozesse wider. Dennoch ist es trotz des Erzielens der hohen theoretischen Sättigung (vgl. Lamnek \& Krell, 2016) aufgrund der geringen Stichprobengröße nicht korrekt, auf die Ansicht einer Grundgesamtheit automatisch rückzuschließen.

Bei der zusammenfassenden qualitativen Inhaltsanalyse wurde die Auswertung von zwei Personen durchgeführt. Dabei überprüften die Autorinnen, ob Textstellen auf die gleiche Weise interpretiert wurden. Somit gewinnt die Deutung an Gewichtung. Trotz gewissenhafter Dokumentation, Zusammenführung und Interpretation der Ergebnisse blieb mit jedem Schritt ein Restrisiko, dass kleine, als unwesentlich betrachtete, jedoch trotzdem wichtige Aussagen auf der Strecke blieben oder dass Anteile verzerrt wahrgenommen wurden.

Praxislehrende haben angesichts der Menge an korrelierenden Anforderungen und den sich ständig ändernden Bedingungen eine äußerst komplexe Aufgabe zu bewältigen (vgl. Schoeneberg, 2014). Deswegen ist es erforderlich, zur multiperspektivischen Erfassung dieses Themas weitere Methoden im Sinne einer Methodentriangulation hinzuzufügen, um die erzielten Ergebnisse zu validieren und/oder um neue Erkenntnisse zu gewinnen.

\section{CONCLUSION}

Die in dieser Arbeit sichtbar gemachten derzeitigen Anforderungen an ergotherapeutische Praxislehrende, welche aufgrund der gemeinsamen gesetzlichen Ausbildungsvorgaben für alle MTD-Berufe zutreffen könnten, sind sehr vielfältig. Diese alle unter Aufrechterhaltung der Qualität der Lehre zu erfüllen und somit die Berufsbefähigung der Absolventinnen und Absolventen sicherzustellen, bedarf eines neuen Stellenwerts für Praxislehrende. Dieses Anforderungsprofil bietet sowohl für die Verantwortlichen an der Fachhochschule als auch für die Lehrenden im Berufsfeld eine wertvolle Grundlage, die Anforderungen bewusst zu reflektieren und die Kompetenzen in diesen Bereichen zu vertiefen.
Für die Praxislehrenden bedeutet das, dass sie, um diese weiten und durchaus tiefen Dimensionen an Anforderungen erfüllen zu können, zusätzlich zu ihrer eigenen Bereitschaft zur Weiterentwicklung auf viele unterstützende Begleitmaßnahmen zurückgreifen können müssen. So wäre es hilfreich,

- wenn die Erstellung eines Rollenbildes für Praxislehrende von den Fachhochschulen und/oder vom Berufsverband in Angriff genommen wird. Bei so komplexen Rollen, wie die Praktikumsanleitung eine darstellt, ist es notwendig, diese zu definieren. Nur so kann sich auch ein Rollenverständnis entwickeln.

- fachhochschulübergreifende Weiterbildungsmaßen für Praxislehrende und daran Interessierte auf Hochschulniveau zu entwickeln und anzubieten.

- eine vermehrte Vernetzung von und intensivere Kommunikation zwischen Praxislehrenden und den Fachhochschulen zu pflegen.

- über das Schaffen von Zeitressourcen durch den Dienstgeber als wichtigen Stakeholder sowohl die strukturellen Rahmenbedingungen für ein Berufspraktikum als auch die Weiterbildung der Praxislehrenden zu unterstützen.

Im Berufsumfeld findet die Weiterentwicklung des Berufsbildes statt, dort wird das theoretische Wissen in die Praxis implementiert. Praxislehrende stehen dabei an vorderster Front, wenn es um einen gelungenen Theorie-Praxis-Transfer geht. Investitionen in deren Aus- bzw. Weiterbildung sind unbedingt notwendig, um den Anspruch, die Studierenden für zukünftige Anforderungen fit zu machen, gerecht zu werden.

\section{DANKSAGUNG}

Die Autorinnen danken den Leitungen des BachelorStudiengangs für Ergotherapie am FH Campus Wien und am Neuromed Campus Linz für die Unterstützung bei der Durchführung der Fokusgruppen sowie den Teilnehmern/-innen der Gruppendiskussion für ihre Teilnahme.
AOTA = American Occupational Therapy Association (2009). SelfAssessment Tool for Fieldwork Educator Competency. Abgerufen am 05.05.2018, von https://www.aota.org///media/Corporate/Files/ EducationCareers/Educators/Fieldwork/Supervisor/Forms/SelfAssessment\%20Tool\%20FW\%20Ed\%20Competency\%20(2009).pdf.

Becker, S. (2009). Wissenschafts- und Forschungskompetenz als Alleinstellungsmerkmal der hochschulischen Bildung? In: Hochschulrektorenkonferenz (Hrsg.), Neue Anforderungen an die Lehre in Bachelor- und Master-Studiengängen, (1)1, 136-140.
Brinker, T. (2009). Die Überprüfung überfachlicher Kompetenzen. In: A. v. Richthofen \& M. Lent (Hrsg.), Qualitätsentwicklung in Studium und Lehre (S. 148-157). Bielefeld: Bertelsmann.

CAOT \& ACOTUP = Canadian Association of Occupational Therapist, \& Association of Canadian Occupational Therapy University Programs (2012). Position Statement: Professional responsibility in fieldwork education in occupational therapy. Abgerufen am 06.05.2018, von: https://caot.in1touch.org/document/3693/F\%20 -\%20Fieldwork\%20Education\%20and\%20OT.pdf. 
DFG = Deutsche Forschungsgemeinschaft (2013). Sicherung guter wissenschaftlicher Praxis. Ergänzte Aufl. Weinheim: Wiley-VCH.

Eberle, S. (2016). Wie lässt sich Identität im Beruf finden? Gazette 6(3), $1-5$.

Ewers, M. (2001). Anleitung als Aufgabe der Pflege. Ergebnisse einer Literaturanalyse. Bielefeld: IPW.

Flick, U. (2011). Triangulation - Eine Einführung. 3., aktualisierte Aufl. Wiesbaden: Springer.

Fink, K. (2014). Qualitätsmanagement an Fachhochschulen - Ein Stakeholder-Ansatz. In: AQ Austria (Hrsg.), Qualitätssicherung zwischen Diversifizierung der Hochschulen und Vereinheitlichung von Standards (S. 53-62). Wien: facultas.

Grunert, C. (2012). Bildung und Kompetenz - Theoretische und empirische Perspektiven auf außerschulische Handlungsfelder. Wiesbaden: Springer.

Grunwald, K. \& Steinbacher, E. (2014). Kompetenz von Leitungskräften - Kompetenzentwicklung durch Leitungskräfte. In: Faas, S., Bauer, P., \& Treptow, R. (Hrsg.), Kompetenz, Performanz, soziale Teilhabe. Sozialpädagogische Perspektiven auf ein bildungstheoretisches Konstrukt (S. 119-138). Wiesbaden: Springer.

Heigl, N. J. (2014). Konflikte verstehen und steuern. Wiesbaden: Springer.

Kellner-Fichtl, M. (2016). Kompetenzanforderungen an Praxisanleiter im Sozialwesen. München: GRIN.

Krinner, M., Kruse-Weber, S. \& Marin, C. (2016). Instrumental- und Gesangspädagogik zwischen Theorie und Praxis. Reflexionen zum ersten Didaktik-Tag an der Kunstuniversität Graz. In: Felden, H. von \& Egger, R. (Hrsg), Qualität in Studium und Lehre - Kompetenzund Wissensmanagement im steirischen Hochschulraum (S. 225242). Wiesbaden: Springer.

Lamnek, S. \& Krell, C. (2016). Qualitative Sozialforschung. 6., überarbeitete Aufl. Weinheim, Basel: Beltz.

Lange, J. (2013). Qualitätsoffensive in der Lehre. In: Claus, S. \& Pietzonka, M. (Hrsg.), Studium und Lehre nach Bologna Perspektiven der Qualitätsentwicklung (S. 47-56). Wiesbaden: Springer.

Lehmann, G. (2017). Lehren mit Erfolg. Renningen: Expert.

Mahler, C., Karsten, S., Roos, M. \& Szecsenyi, J. (2012). Interprofessionelle Ausbildung für eine patientenzentrierte Versorgung der Zukunft. Die Entwicklung eines Kompetenzprofils für den Bachelor-Studiengang „Interprofessionelle Gesundheitsversorgung“. Zeitschrift für Evidenz, Fortbildung und Qualität im Gesundheitswesen 106(7), 523-532.

Mamerow, R. (2016). Praxisanleitung in der Pflege. 5., aktualisierte Aufl. Berlin Heidelberg: Springer.

Markowitsch, J. (2001). Praktisches akademisches Wissen. Wien: Universitätsverlag.

Martens, M. \& Asbrand, B. (2009). Rekonstruktion von Handlungswissen und Handlungskompetenz - auf dem Weg zu einer qualitativen Kompetenzforschung. Zeitschrift für Qualtiative Forschung 10(2), 201-217.
Mayring, P. (2015). Qualitative Inhaltsanalyse - Grundlagen und Techniken. 12., überarbeitete Aufl. Weinheim, Basel: Beltz.

Perkhofer-Czapek, M. \& Potzmann, R. (2016). Begleiten, Beraten und Coachen - Der Lehrberuf im Wandel. Wiesbaden: Springer.

Pfäffli, B. (2015). Lehren an Hochschulen. 2. Aufl. Göttingen: Die Werkstatt.

Preyer, G. (2012). Rolle, Status, Erwartungen und soziale Gruppe - Mitgliedschaftstheoretische Reinterpretationen. Wiesbaden: Springer.

Schoch, R. (2016). Warum nicht Fokusgruppen? Abgerufen am 19.04.2017, von http://www.wiso-schoch.ch/fr_dt/00_ AKTUELL/2016/06_2016/Focus\%20Groups.pdf.

Schoeneberg, K. (2014). Komplexität - Einführung in die Komplexitätsforschung und Herausforderungen für die Praxis. In: Schoeneberg, K. (Hrsg). Komplexitätsmanagement in Unternehmen (S. 13-28). Wiesbaden: Springer Gabler.

Schulz, M. (2012). Quick and easy!? Fokusgruppen in der angewandten Sozialwissenschaft. In: Schulz, M., Mack, B. \& Renn, O. (Hrsg.), Fokusgruppen in der empirischen Sozialwissenschaft (S. 9-22). Wiesbaden: Springer.

Seithe, M. (2012). Schwarzbuch soziale Arbeit. Wiesbaden: Springer. Strobel, A. \& Westhoff, K. (2010). Eignungsinterview. In: K. Westhoff, C. Hagemeister, M. Kersting, F. Lang, H. Moosbrugger, G. Reimann \& G. Stemmler (Hrsg.), Grundwissen für die berufsbezogene Eignungsbeurteilung nach DIN 33430 (S. 84-101). Lengerich, Berlin, Bremen, Miami, Riga, Viernheim, Wien, Zagreb: Papst Science.

Tong, A., Sainsbury, P. \& Craig, J. (2007). Consolidated criteria for reporting qualitative research (COREQ): a 32-item checklist for interviews and focus groups. International Journal for Quality in Health Care 19(6), 349-357. Abgerufen am 12.07.2018, von https://doi.org/10.1093/intqhe/mzm042.

Von der Heyden, R. (2013). Berufliche Schlüsselprobleme als Grundlage einer kompetenzorientierten Fachdidaktik Ergotherapie. Abgerufen am 18.09.2017, von http://www.bwpat.de/ausgabe24/ heyden_bwpat24.pdf.

Webler, W. (2004). Lehrkompetenz - über eine komplexe Kombination aus Wissen, Ethik, Handlungsfähigkeit und Praxisentwicklung. Bielefeld: UVW.

Weinert, A. B. (2004). Arbeits- und Organisationspsychologie. Weinheim: Beltz.

WMA = World Medical Association (2013). Abgerufen am 25.05.2018, von: http://www.bundesaerztekammer.de/fileadmin/user_upload/ Deklaration_von_Helsinki_2013_DE.pdf.

Wright, J. M. (2009). The Frameworks: Implications for Placement Learning. In: A. Smith, H. Mc Askill \& K. Jack (Hrsg.), Developing Advanced Skills in Practice Teaching (S. 6-14). London, Hampshire, New York: Palgrave Macmillan. 